\title{
Ripple Reduction Characteristics in the Parallel-Connected DC Power Distribution System Constructed by New Terminals for Wire Communication
}

\author{
Akihiko Katsuki Member (Kyushu Institute of Technology, katsuki@cse.kyutech.ac.jp) \\ Tadashi Matsumoto Member (Matsushita Electric Works, Ltd., matumoto@mail.mew.co.jp) \\ Shogo Watanabe Non-member (Kyushu Institute of Technology, watanabe@ scorpio5.cse.kyutech.ac.jp) \\ Masaichi Fukunaga Non-member (Matsushita Electric Works, Ltd., fukunaga@mail.mew.co.jp)
}

Keywords: electronic choke, powering terminal, powered terminal, voltage-ripple attenuation, wire communication system

In most wire communication systems, communication lines are also utilized as dc power lines. So far, we proposed a new wire communication system. Main part of this system consists of lowpower switched-mode dc-dc converters. These converters construct a parallel connected power supply. Reliability and availability of the proposed wire communication system is higher than the conventional wire communication system. In this system, we considered two types of terminals, i.e., "Powering terminal" and "Powered terminal." Powering terminal is powered by commercial ac and supplies dc power to the communication lines. Powered terminal is powered from the communication lines.

Powering terminal should prevent signal inflow from the communication lines. Powered terminal in most wire communication systems needs to separate ac signals and dc supply power in its input part connected to the communication lines. Therefore, the high input impedance circuit is needed in the input part of each terminal. The power loss of a conventional electronic choke circuit with high input impedance is high, and the direction of dc supply power is unidirectional. In our system, the direction of dc power is opposite in powering terminal at reception and powered terminal. Therefore, to use the same electronic choke for powering terminal and powered terminal, it is preferable that the electronic choke be bidirectional. So, we have proposed new electronic choke by the use of small inductors and an amplifier. The electronic choke has high impedance and an ability of good attenuation against signals. In addition, it has extremely low generation of own noise.

For example, we consider about two buck-type dc-dc converters connected in parallel as a simple model. And, electronic chokes are connected as shown in Fig. 1(a). Here, switching frequencies of the converters were $200 \mathrm{kHz}$ and $210 \mathrm{kHz}$. Drive signals for switching devices were not synchronized and their duty ratios were set to 0.5 . The observation waveform of the output ripple voltage across the load resistor is shown in Fig. 1(b). This observation waveform is near the noise of our measurement system. We consider about two converters without electronic chokes connected in parallel for the comparison. Fig. 2 is observation waveform of the output ripple voltage of two converters connected in parallel. We can observe periodic beat with the frequency of $10 \mathrm{kHz}$.

The electronic choke is a circuit of high performance in the attenuation against signal. The electronic choke can almost remove voltage ripple from the converter. This electronic choke can be also used in conventional wire communication system.

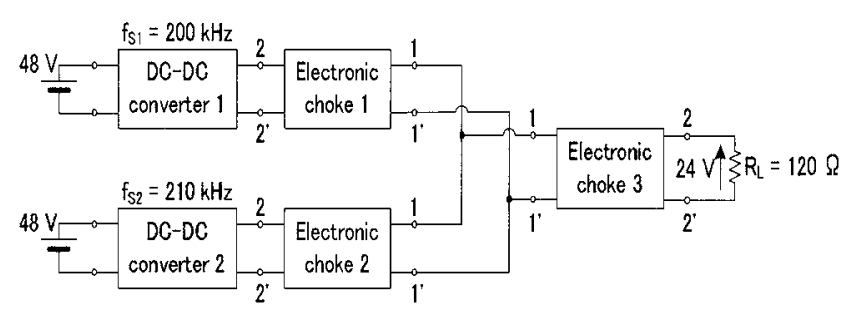

(a) Experimental circuit

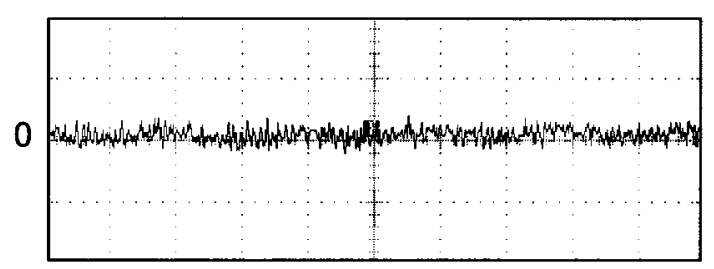

(b) Observed waveform of the output ripple voltage across the load resistor $\mathrm{R}_{\mathrm{L}}$ (Scale: $20 \mathrm{mV} / \mathrm{div}, 50 \mu \mathrm{s} / \mathrm{div}$ )

Fig. 1. Output ripple voltage of the parallel-connected dc-dc converters with the electronic chokes

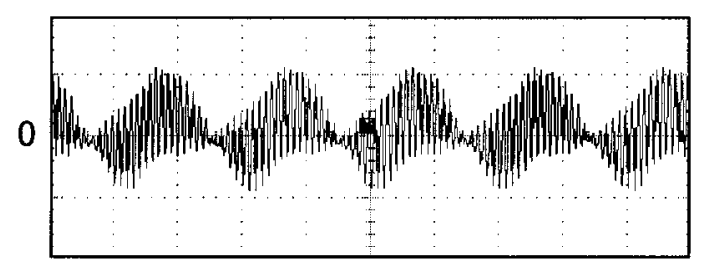

Fig. 2. Output ripple voltage of the basic parallelconnected dc-dc converters removing the electronic chokes from Fig. 1(a). Observed waveform of the output ripple voltage across the load resistor $\mathrm{R}_{\mathrm{L}}$ (Scale: $200 \mathrm{mV} / \mathrm{div}, 50 \mu \mathrm{s} / \mathrm{div})$ 


\title{
Ripple Reduction Characteristics in the Parallel-Connected DC Power Distribution System Constructed by New Terminals for Wire Communication
}

\author{
Akihiko Katsuki* Member \\ Tadashi Matsumoto** Member \\ Shogo Watanabe* Non-member \\ Masaichi Fukunaga $^{* *} \quad$ Non-member
}

\begin{abstract}
Powering terminal should prevent signal inflow from the communication lines. Powered terminal in most wire communication systems needs to separate ac signals and dc supply power in its input part connected to the communication lines. Our original communication system consists of low-power dc-dc converters. In our system, the direction of dc power is opposite in powering terminal at reception and powered terminal. Therefore, to use the same electronic choke for powering terminal and powered terminal, it is preferable that the electronic choke be bidirectional. So, we have proposed new electronic choke by the use of small inductors and an amplifier. From the viewpoint of power distribution, this system contains a parallel-connected dc power supply. In this paper, voltage-ripple attenuation characteristics at reception are presented on the dc power distribution system using buck-type converters and new electronic chokes. New electronic choke has an ability of good attenuation against signals. Even though switching frequencies are different, no beat is observed in the output dc voltage waveform of powered terminal. New electronic choke effectively removes circulation or interference by the ripple.
\end{abstract}

Keywords: electronic choke, powering terminal, powered terminal, voltage-ripple attenuation, wire communication system

\section{Introduction}

In most wire communication systems, communication lines are also utilized as dc power lines ${ }^{(1)}$. Usually, a main power unit supplies all the power consumed in input/output terminal devices. These systems have common problems in reliability and availability. Moreover, the adaptability to alterations is low in the system. When the power capacity is insufficient by increase of the number of terminals, it is necessary to exchange the main power-supply. If the main powersupply stops operating, the whole system breaks down.

To remove these disadvantages, we proposed a new wire communication system $^{(2)}$. Main part of this system is constructed by using low-power switched-mode dc-dc converters. A converter is utilized as power supply having the function of transmitter. The transmission of signal is performed by modulating the output ripple voltage. These converters construct a parallel-connected power supply. When the total power rating of converters is selected to be more than that of the terminal devices powered from the communication lines, this combination makes a parallel redundant system. The system reliability and availability are much increased in comparison with conventional wire communication systems. When the total power capacity is insufficient by increase of the number of terminals, the total power capacity can be easily

\footnotetext{
* Kyushu Institute of Technology

680-4, Kawazu, Iizuka-shi, Fukuoka 820-8502

** Matsushita Electric Works, Ltd.

1048, Kadoma, Osaka 571-8686
}

increased by newly connecting the power supply. Failure in small number of terminals leads to no system down. Repair or maintenance can be implemented with the system active. This system is suitable for controlling electronic equipment and others. We have already presented signal transmission by the way of analog or digital AM (Amplitude Modulation $)^{(2)-(4)}$. In “(5)", characteristics of modulation and demodulation by digital FM (Frequency Modulation) were shown. To realize free-topology connection to communication lines, we introduced a new electronic choke ${ }^{(5)}$. It has high impedance against ac signal and an excellent ability of ac component attenuation ${ }^{(6)}$. And, it can separate ac communication signals and dc supply power.

The powering terminals in our system make parallelconnected dc power supply. In this paper, voltage-ripple reduction characteristics at the state of reception are presented on the dc power distribution system using buck-type PWM (Pulse Width Modulation) dc-dc converters and new electronic chokes. It is described that voltage-ripple of periodic beat by a parallel-connected dc power supply can be almost removed by the new electronic choke.

\section{System Configuration and Operation}

Configuration of the proposed system is shown in Fig. 1. We considered two types of terminals, i.e., "Powering terminal" and "Powered terminal." Fig. 2 and Fig. 3 explain the operation of powering terminal and powered terminal, at reception, respectively. About switches, "R" and " $T$ " represent "Reception" and "Transmission" respectively. All powering 
terminals have dc-dc converter of low power rating. At transmission of powering terminal, output ripple voltage is intentionally increased and modulated by the signal. The communication signal frequency is basically equal to the switching frequency $f_{\mathrm{s}}$. In addition, it has controller, receiver, and electronic choke. The electronic choke is described in the next chapter. Uninterruptible power supply (UPS) is placed between the commercial ac lines and dc-dc converter against commercial ac power failure. Powering terminal is powered

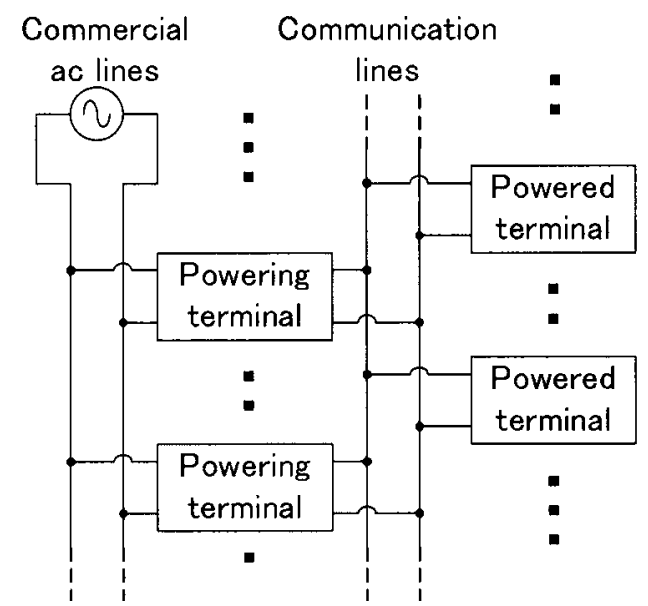

Fig. 1. Configuration of the proposed wire communication system by the commercial ac and supplies dc power to the communication lines. Powered terminal is powered from the communication lines.

In the powering terminal at reception, the power-supply current "DC" flows toward terminals $1-1$ ' from terminals 22 ' of the electronic choke. The communication signal "AC" is supplied from terminals $1-1$ ' of the electronic choke. The direction of the dc power is opposite in the state of transmission and reception. In the powered terminal at reception, the power-supply current "DC" and communication signal "AC" are supplied together from terminals $1-1$ ' of the electronic choke. And, the communication signal "AC" is taken out of terminals $3-3^{\prime}$ of the electronic choke.

\section{New Electronic Choke}

Against signals, all terminals are parallel-connected ac load. In order to connect many terminals to the communication lines, input impedance of each terminal needs to be high. There is a conventional electronic choke circuit as one of methods of increasing input impedance ${ }^{(7)}$.

This electronic choke circuit utilizes high output impedance of a common-base bipolar transistor at active state. The dc power dissipation increases when the load current is high. In addition, the dc current is limited to unidirectional flow. This circuit was proposed for the wire telephone system. It is assumed to use with constant-current power supply.

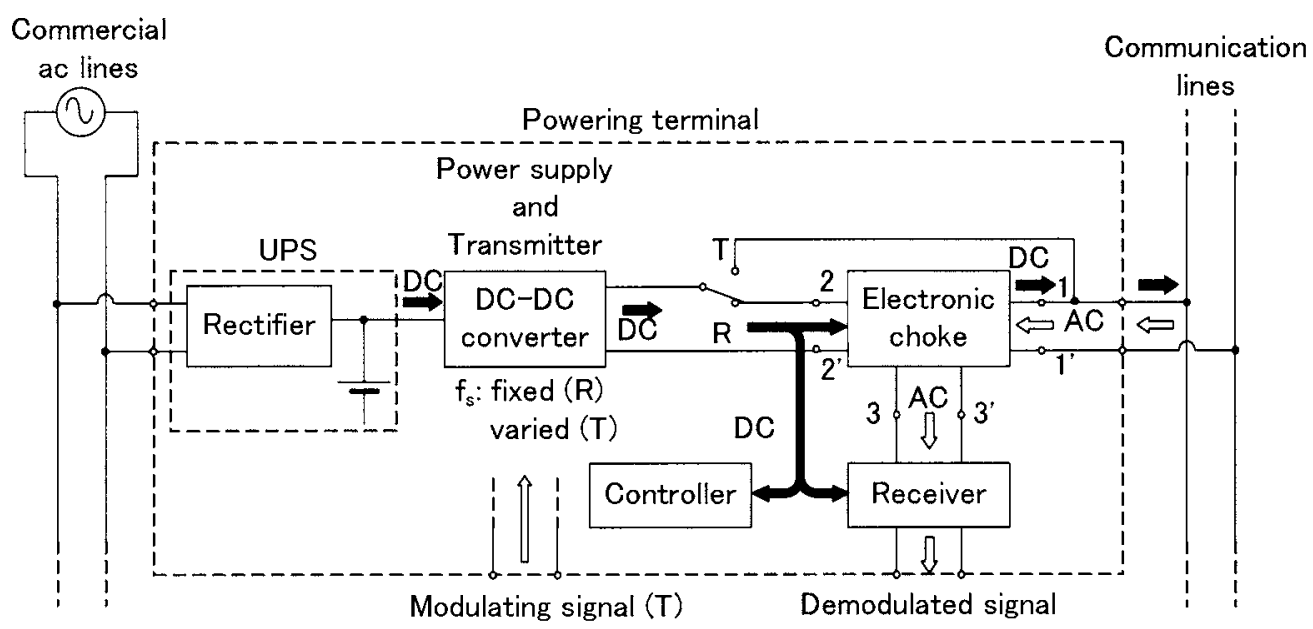

Fig. 2. Operation of powering terminal in the proposed wire communication system

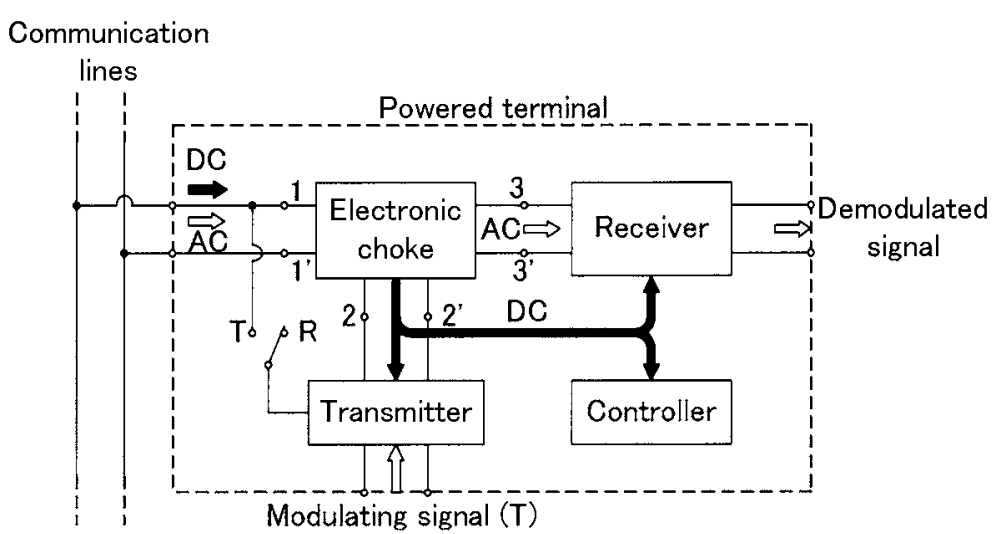

Fig. 3. Operation of powered terminal in the proposed wire communication system 
In the powering terminal of our original system, it is preferable that electronic choke be bidirectional. So, we introduced low-loss new electronic choke (hereafter, it's called the electronic choke) with small inductors and an amplifier ${ }^{(5)}$. The electronic choke increases input impedance that looked at each terminal from the communication lines. Therefore, it becomes easy to put the signal on the communication lines. Moreover, it also has working that separates communication signal and dc supply power.

3.1 Impedance Characteristics Fig. 4(a) shows input impedance measurement circuit of the electronic choke. For example, when winding turns ratio of the inductor $\mathrm{L}_{\mathrm{M}}$ is $1: 1$ and voltage gain $A$ of the amplifier is selected to 2 , the input impedance $Z_{\text {in }}$ looking into terminals $1-1^{\prime}$ becomes very high. Measured data on the absolute value $\left|Z_{\text {in }}\right|$ is presented in Fig. 4(b), where inductance $L_{\mathrm{B}}, L_{\mathrm{M}}$, input impedance of the amplifier, dc power supply voltage $V_{\text {in }}$, and dc load current are $0.5 \mathrm{mH}, 2 \mathrm{mH}, 100 \mathrm{k} \Omega, 24 \mathrm{~V}$, and $0.1 \mathrm{~A}$, respectively. In Fig. 4(b), $\left|Z_{\text {in }}\right|$ shows the value beyond about $50 \mathrm{k} \Omega$ between two resonant frequencies. This value is higher than impedance of the inductors though it is parallel with the amplifier. And $Z_{\text {in }}$ becomes near resistive in the range between two resonant frequencies ${ }^{(5)}$. Resistive impedance is desirable for realizing free-topology connection of terminals. It is preferable to communicate in the frequency range between these two peaks. The capacitances $C_{1}, C_{2}$, and $C_{3}$ were selected to have no influence on the frequency between two peaks. The lower cutoff frequency of the amplifier is decided with $C_{1}$ and $C_{3}$. And, $C_{2}$ was decided for impedance to become small enough against the ripple voltage.

Necessary value of the input impedance depends on the reception driver. We assume that the terminal can be connected until that parallel impedance of the terminals becomes equal to the characteristics impedance of the communication lines. In example of practical, terminals of about 250 per one system are connected in a usual office building. The characteristics impedance of the low cost communication lines are about $80 \Omega$. Therefore, we consider that necessary input impedance for practical use is about $20 \mathrm{k} \Omega$ at the minimum.

\subsection{AC Component Attenuation Characteristics}

The electronic choke needs to separate signals and dc supply power. It is desirable for ac component not to appear in dc output. The ac component attenuation characteristics from terminals $1-1^{\prime}$ to $2-2^{\prime}$ are shown in Fig. 5. These assume powering terminal of transmission and powered terminal. The ac component attenuation characteristics from terminals $2-2^{\prime}$ to $1-1^{\prime}$ are shown in Fig. 6. These assume powering terminal of reception.

Fig. 5(a) and Fig. 6(a) are experimental circuits of ac component reduction ratio. Fig. 5(b) and Fig. 6(b) are measurement data of ac output voltage $v_{\text {out }}$ divided by ac input voltage $v_{\text {in }}$. When $v_{\text {out }} / v_{\text {in }}$ was graphed, decibel expression was used and it is named voltage gain $G_{\mathrm{v}}$. The ac component reduction ratio from terminals $1-1^{\prime}$ to $2-2^{\prime}$ is a value enough for

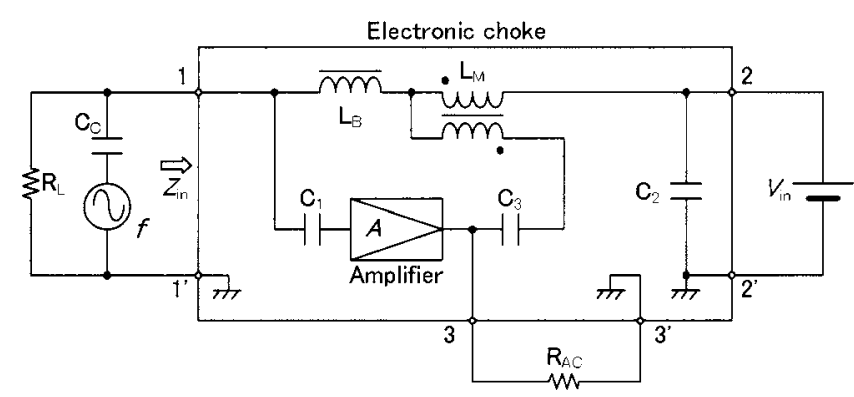

(a) Experimental circuit

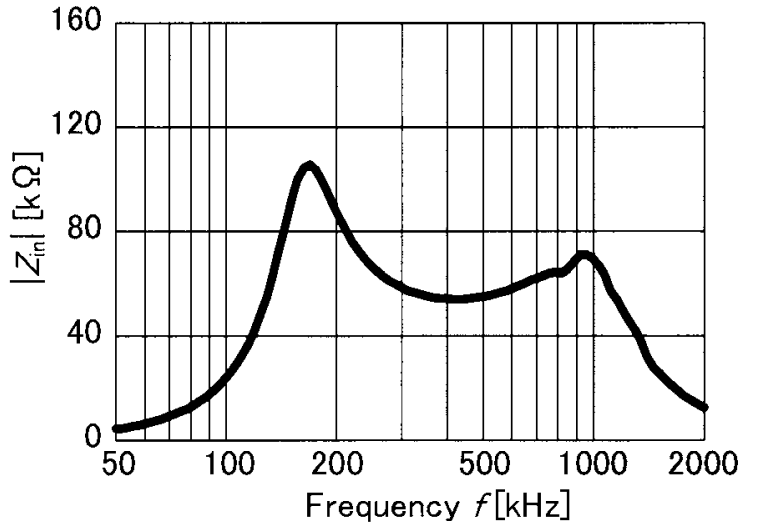

(b) Measured data

Fig. 4. Frequency characteristics of the input impedance $Z_{\text {in }}$ in the electronic choke, where, $C_{1}=0.1 \mu \mathrm{F}, C_{2}=C_{3}=1 \mu \mathrm{F}$

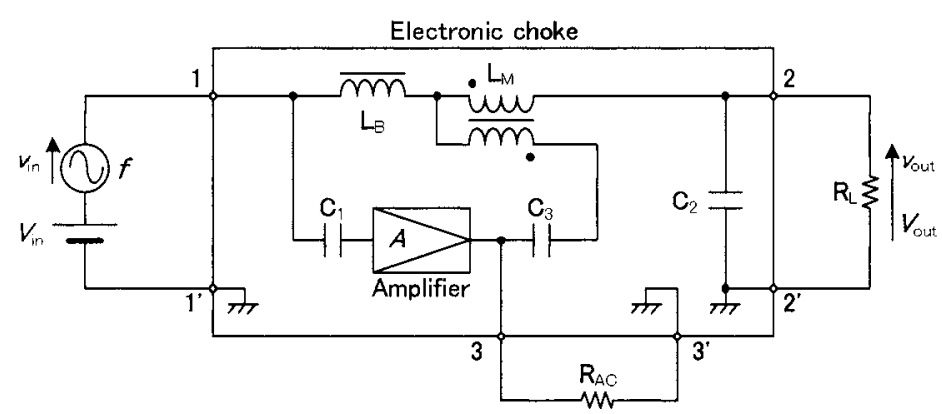

(a) Experimental circuit

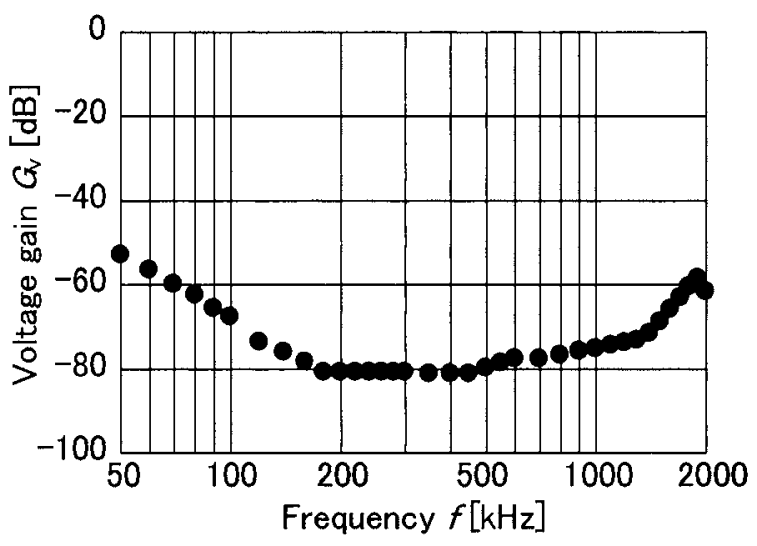

(b) Measured data

Fig. 5. AC component reduction characteristics from 1-1' to $2-2^{\prime}$ in the electronic choke 
practical use. We consider that enough if ac voltage attenuated up to $5 \%$ referring to the tolerance of the power-supply voltage of a usual digital logical integrated circuit. In this time, because the dc output voltage assumes $24 \mathrm{~V}, 1.2 \mathrm{~V}_{\mathrm{p}-\mathrm{p}}$ is a tolerance in the ac voltage of the dc output terminals. For example, if only $-26 \mathrm{~dB}$ is attenuated when the maximum ac signal of $24 \mathrm{~V}_{\mathrm{p}-\mathrm{p}}$ is supplied, it is enough. However, the attenuation of $-70 \mathrm{~dB}$ or more was obtained in the electronic choke. Fig. 6(b) is compared with Fig. 5(b) and the effect of attenuation is small. However, the ac component supplied to terminals 2-2' doesn't need high attenuation, because it corresponds to voltage-ripple of the dc power supply and it is small by nature.

3.3 Noise Characteristics The noise characteristics of the electronic choke was investigated by experimental circuit of Fig. 7(a) and Fig. 8(a). The power consumption of the powered terminal is supposed several watts in the system that we are assuming now. Fig. 7(b) and Fig. 8(b) are the observed waveforms of the noise that appear to terminals $2-2^{\prime}$ and terminals $1-1^{\prime}$ of the electronic choke, respectively. When the observed waveforms are compared with app.Fig. 1 of appendix, we can see that these voltages are almost same with noise voltage in our measurement system. Therefore, it seems that the electronic choke doesn't putout the noise so

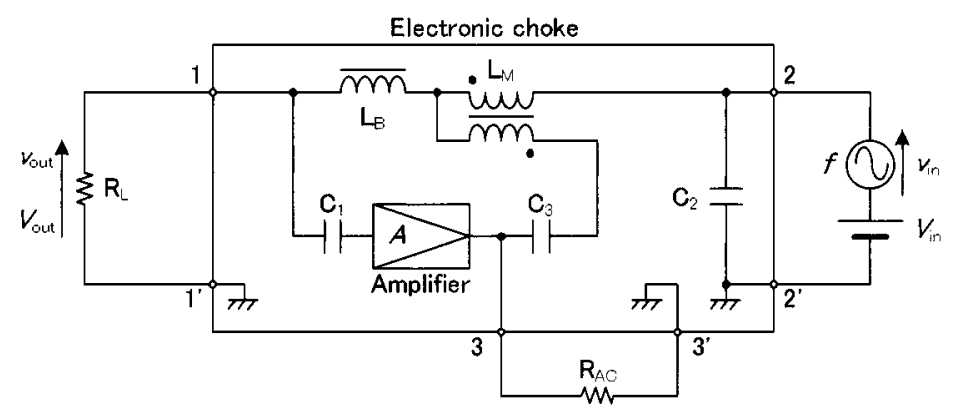

(a) Experimental circuit

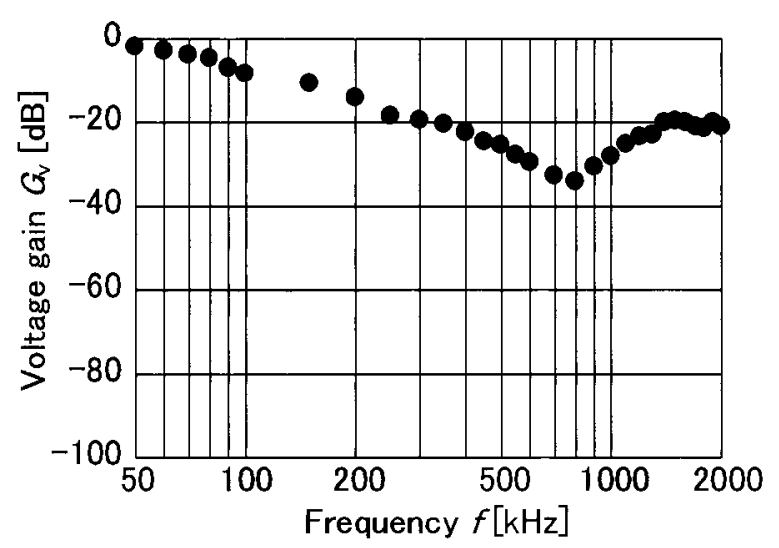

(b) Measured data

Fig. 6. AC component reduction characteristics from $2-2^{\prime}$ to $1-1^{\prime}$ in the electronic choke

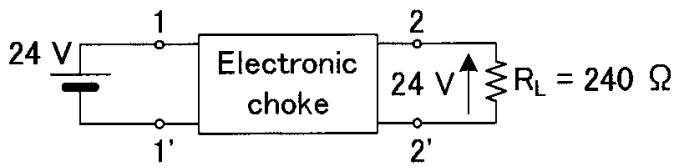

(a) Experimental circuit (DC voltage is supplied from 1-1')

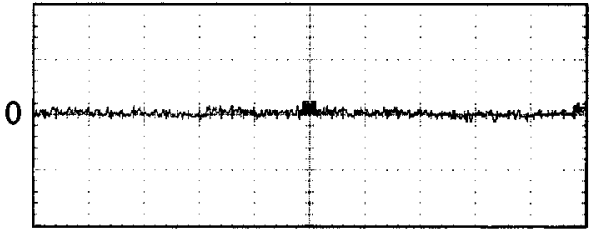

(Scale: $10 \mathrm{mV} / \mathrm{div}, 5 \mu \mathrm{s} / \mathrm{div}$ ).

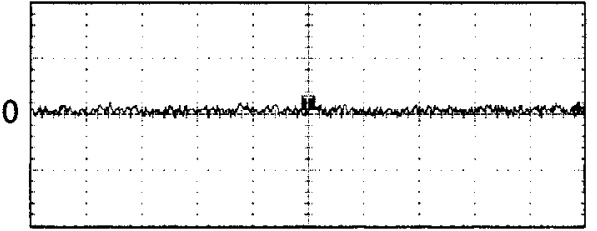

(Scale: $10 \mathrm{mV} / \mathrm{div}, 50 \mu \mathrm{s} / \mathrm{div}$ ).

(b) Observed ac voltage waveform across 2-2'

Fig. 7. Noise voltage waveform that appears to the terminals $2-2^{\prime}$ of the electronic choke

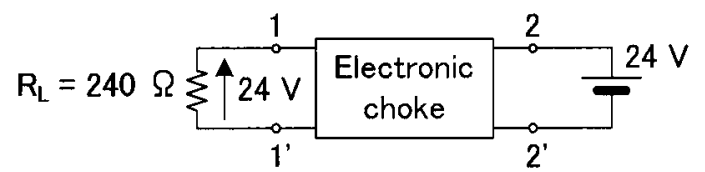

(a) Experimental circuit (DC voltage is supplied from 2-2')

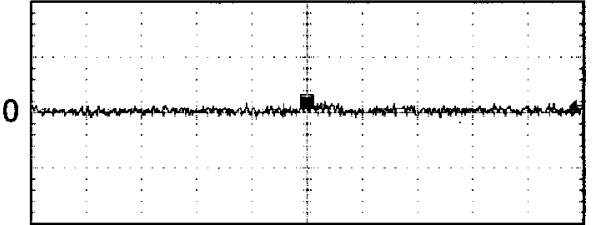

(Scale: $10 \mathrm{mV} / \mathrm{div}, 5 \mu \mathrm{s} / \mathrm{div}$ ).

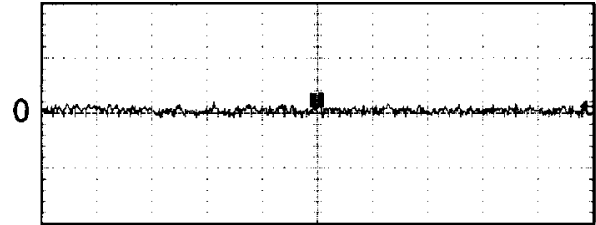

(Scale: $10 \mathrm{mV} / \mathrm{div}, 50 \mu \mathrm{s} / \mathrm{div}$ ).

(b) Observed ac voltage waveform across 1-1'

Fig. 8. Noise voltage waveform that appears to the terminals $11^{\prime}$ of the electronic choke 
much. The comparator having hysteresis is usually built into the receiver for a digital signal. Because the noise voltage is small, the width of hysteresis can be narrowed. It is advantageous against the phase delay. In this result, the phase delay was able to be suppressed enough, and the real time system was obtained.

\section{Ripple in Parallel-Connected DC Power Supply}

In this experiment, two buck-type PWM dc-dc converters are used for the power supply to the communication lines. Their duty ratios were set to 0.5 . These converters make the $24-\mathrm{V}$ dc from the 48-V dc. No noise filter is applied, though simple snubber circuits are applied to the switching devices.

The output ripple voltage of this converter was examined. The switching frequency $f_{\mathrm{s}}$ of converter was set to $200 \mathrm{kHz}$

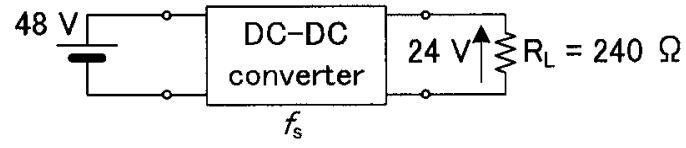

(a) Experimental circuit

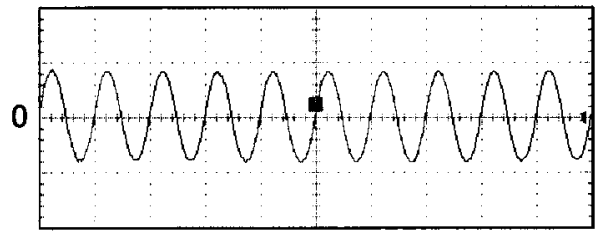

(b) Observed waveform of the output ripple voltage across the load resistor $\mathrm{R}_{\mathrm{L}}$, when $f_{\mathrm{s}}=200 \mathrm{kHz}$ (Scale: $200 \mathrm{mV} / \mathrm{div}, 5 \mu \mathrm{s} / \mathrm{div}$ )

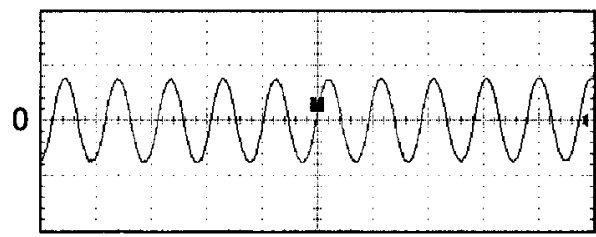

(c) Observed waveform of the output ripple voltage across the load resistor $\mathrm{R}_{\mathrm{L}}$, when $f_{\mathrm{s}}=210 \mathrm{kHz}$ (Scale: $200 \mathrm{mV} /$ div, $5 \mu \mathrm{s} /$ div)

Fig. 9. Output ripple voltage that appears to load resistor of the converter or $210 \mathrm{kHz}$. The output ripple voltage waveform is observed with the experimental circuit shown in Fig. 9(a). The observation waveforms of $200 \mathrm{kHz}$ and $210 \mathrm{kHz}$ in $f_{\mathrm{s}}$ are shown in Fig. 9(b) and Fig. 9(c), respectively.

Fig. 10(a) shows an example of basic parallel connection by two buck-type PWM dc-dc converters as the simplest model. The switching frequency $f_{\mathrm{s} 1}$ of the converter 1 and the switching frequency $f_{\mathrm{s} 2}$ of the converter 2 are $200 \mathrm{kHz}$ and $210 \mathrm{kHz}$, respectively. The switching signal is a square wave of duty ratio 0.5 . Drive signals for switching devices were not synchronized. In Fig. 10(b), we can observe periodic beat with the frequency of $10 \mathrm{kHz}$ in the output ripple voltage waveform. It can be surmised that this frequency of $10 \mathrm{kHz}$ is the difference of the frequency of the output ripple voltage of each converter.

The terminals $1-1^{\prime}$ of the electronic choke built into each terminal are connected to the communication lines. Therefore, terminals $1-1^{\prime}$ actually corresponds to the communication line. Fig. 11(a) is connected example of the electronic

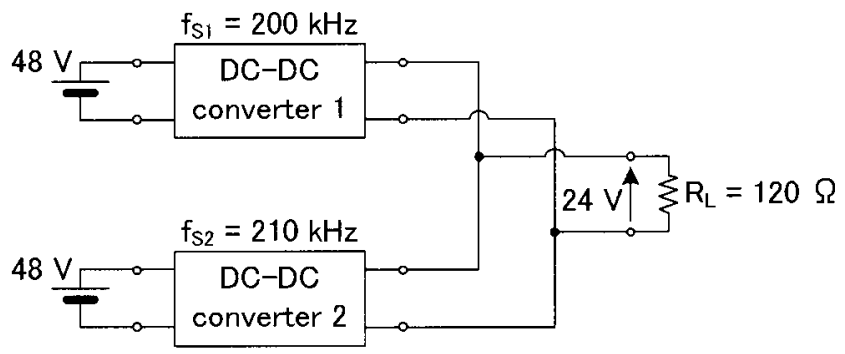

(a) Experimental circuit

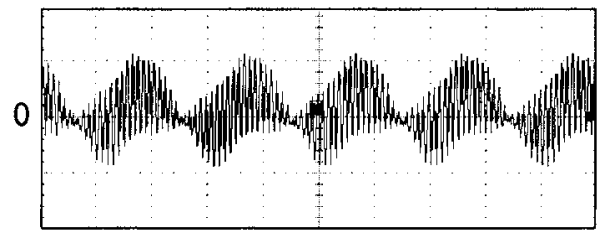

(b) Observed waveform of the output ripple voltage across the load resistor $\mathrm{R}_{\mathrm{L}}$ (Scale: $200 \mathrm{mV} /$ div, $50 \mu \mathrm{s} /$ div)

Fig. 10. Output ripple voltage of the basic two parallelconnected dc-dc converters

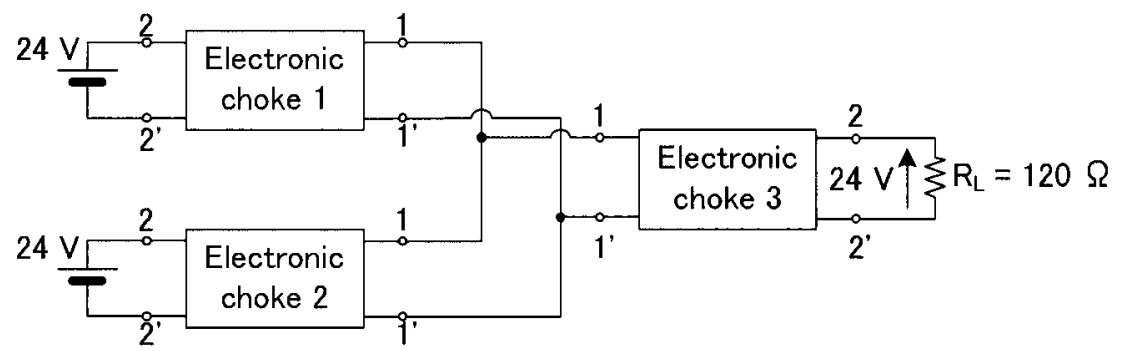

(a) Experimental circuit

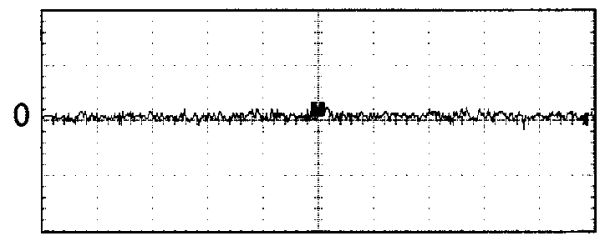

(b) Observed voltage waveform across the load resistor $\mathrm{R}_{\mathrm{L}}$ (Scale: $10 \mathrm{mV} / \mathrm{div}, 50 \mu \mathrm{s} / \mathrm{div}$ )

Fig. 11. Output noise voltage of two stabilized power supplies with the electronic chokes 


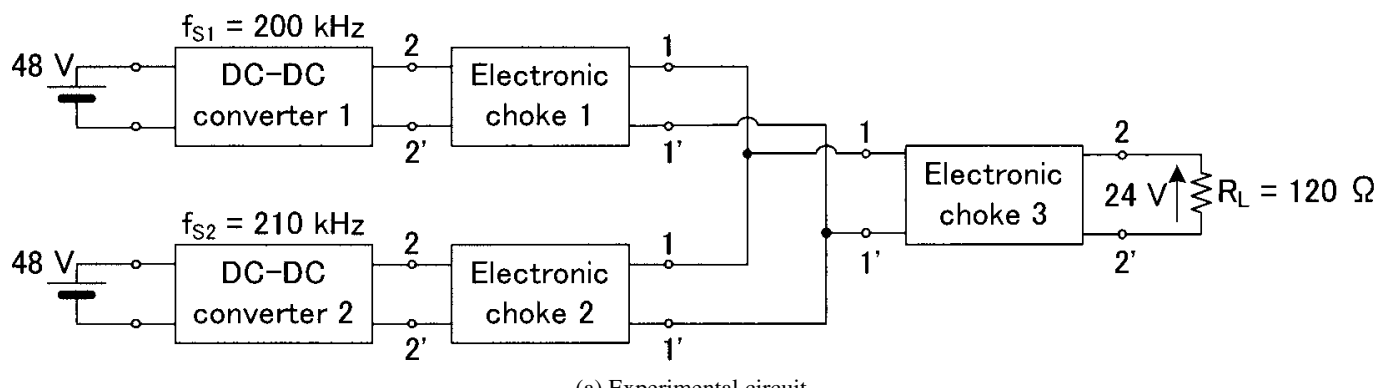

(a) Experimental circuit

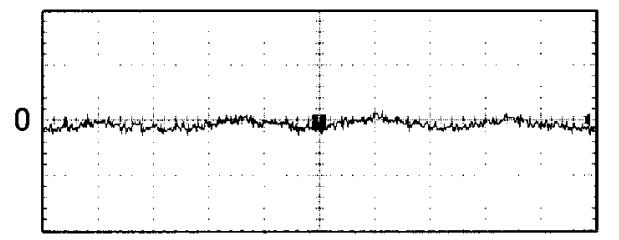

(b) Observed waveform of the output ripple voltage across the load resistor $\mathrm{R}_{\mathrm{L}}$ (Scale: $20 \mathrm{mV} / \mathrm{div}, 50 \mu \mathrm{s} / \mathrm{div}$ )

Fig. 12. Output ripple voltage of the parallel-connected dc-dc converters with the electronic chokes

choke of the simplest model. Fig. 11(b) is observation waveform of the noise that appears to the load resistor. When the observation waveform is compared with the app.Fig. 1 of appendix, these seem to be almost same with noise in our measurement system. Therefore, it seems that the electronic choke doesn't putout the noise by the connecting method of Fig. 11(a).

In Fig. 12(a), the converters are connected with the electronic choke 1 and 2 instead of stabilized power supplies. This circuit is equivalent to two powering terminals and one powered terminal at reception. The converters are connected to the terminals $2-2^{\prime}$ of the electronic chokes 1 and 2 . The electronic choke 3 is assumed powered terminal, and dc load resistor is connected to terminals $2-2^{\prime}$. Terminals $1-1^{\prime}$ of the electronic choke correspond to connecting points with the communication lines. The switching frequency $f_{\mathrm{s} 1}$ of converter 1 and the switching frequency $f_{\mathrm{s} 2}$ of converter 2 are $200 \mathrm{kHz}$ and $210 \mathrm{kHz}$ respectively as Fig. 10. The observation waveform of the output ripple voltage across the load resistor is shown in Fig. 12(b). The electronic choke has an ability of good attenuation from terminals $1-1^{\prime}$ to $2-2^{\prime}$ and from terminals $2-2^{\prime}$ to $1-1^{\prime}$. Against signals of $200 \mathrm{kHz}$ or $210 \mathrm{kHz}$, measured data were about $-80 \mathrm{~dB}$ from terminals 1 $1^{\prime}$ to $2-2^{\prime}$ and about $-16 \mathrm{~dB}$ from terminals $2-2^{\prime}$ to $1-1^{\prime}$. The periodic beat of $10 \mathrm{kHz}$ shown in Fig. 10(b) becomes small by using the electronic choke.

A conventional electronic choke circuit is used on the assumption of the constant-current power supply. On the other hand, the proposed electronic choke is used on the assumption of the constant-voltage power supply. The system configuration such as Fig. 11 and Fig. 12 is advantageous from the viewpoint of safety with a proposed electronic choke. Moreover, the proposed electronic choke can use the same circuit as the powering terminal and the powered terminal.

\section{Conclusion}

Fundamental ripple characteristics of our wire communication system were investigated by experiments. The electronic choke is a circuit of high performance in the attenuation against signal. The electronic choke can almost remove voltage-ripple from the converter. Moreover, when the switching frequency of each converter is different, the periodic beat can be also removed by the electronic choke. In our original communication system, the electronic choke is built into each terminal. The electronic choke has extremely low generation of own noise. And, the interference with output ripple voltage of converter can be removed. This electronic choke can be also used in conventional wire communication systems.

(Manuscript received May 11, 2005,

revised March 21, 2006)

This paper was presented at IPEC-Niigata 2005, and approved for publication in the IEEJ Transactions on Industry Applications Society.

\section{References}

( 1 ) For example, H. Ott and H. Zierhut: “Übertragungssystem”, Europäische Patentschrift, 365696B1 (1995-2)

( 2 ) A. Katsuki, M. Matsushima, and N. Takimoto: "Wire Communication System Utilizing Ripple-Modulated DC-DC Converter as Signal Transmitter and Power Supply", Proc. IEEE INTELEC '99, No.13-1 (1999-6)

( 3 ) A. Katsuki, S. Ogura, and M. Matsushima: "Ripple Characteristics against Inductive Load of a High-ESR-Filter-Capacitor DC-DC Converter Utilized in New Wire Communication System”, Proc. IEEJ IPEC '00, pp.108-117 (2000-4)

( 4 ) A. Katsuki and M. Matsushima: "Signal Transmission Characteristics of Inductively Loaded High-Ripple DC-DC Converter Used in a New Wire Communication System”, Proc. IEEE INTELEC '00, pp.741-748 (2000-9)

( 5 ) A. Katsuki, T. Matsumoto, T. Eto, and Y. Hashimoto: "Digital-FM Transmission and Reception in a New Wire-Communication System That Utilizes DC-DC Converter as Transmitter", Proc. IEEE INTELEC '03, pp.615-622 (2003-10)

(6) A. Katsuki and T. Matsumoto: "AC Voltage Attenuation Characteristics of a New Electronic Choke Used in the Terminal of a Wire Communication System", J. of the Magnetics Soc. of Japan, Vol.28, No.4, pp.620-626 (2004-4) (in Japanese)

( 7 ) S. Nojima, N. Ooba, and K. Yamamoto: "Electronic Choke Circuit", Japanese Patent, 2079176 (1995-8) (in Japanese) 


\section{Appendix}

For the reference, the voltage waveforms when the probe of the oscilloscope is short-circuited are shown in app.Fig. 1. These show the lowest level of the noise in our measurement system.

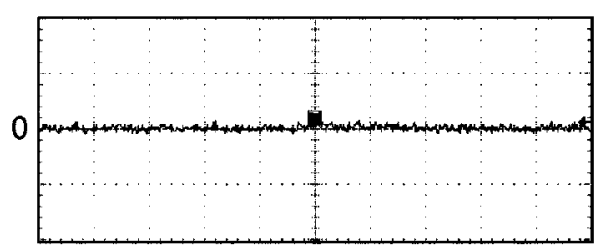

(Scale: $10 \mathrm{mV} / \mathrm{div}, 5 \mu \mathrm{s} / \mathrm{div}$ ).

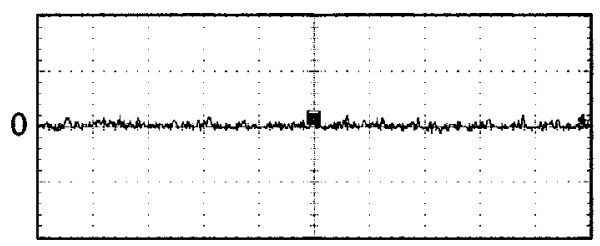

(Scale: $10 \mathrm{mV} / \mathrm{div}, 50 \mu \mathrm{s} / \mathrm{div}$ ).

app. Fig. 1. Observed noise voltage waveform when probe of oscilloscope is short-circuited

Akihiko Katsuki (Member) was born in Fukuoka, Japan, in 1956.

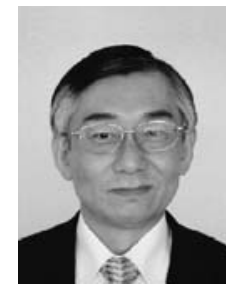

He received his B. E., M. E., and Dr. Eng. degrees from Kyushu University in 1978, 1980, and 1983, respectively. He is presently an associate professor at Kyushu Institute of Technology. He has worked on nonlinear magnetics, power electronics, and communication circuit. Institute of Electronics, Information and Communication Engineers of Japan, Magnetics Society of Japan, Institute of Electrical and Electronics Engineers member.

Tadashi Matsumoto (Member) was born in Shimane, Japan, in 1979

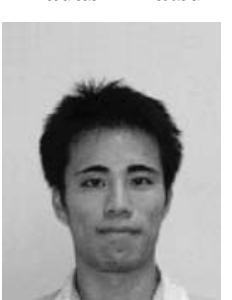
He received his B. S. and M.S. degrees in information engineering from the Kyushu Institute of Technology, Fukuoka, in 2002 and 2004, respectively. He is an employee in Matsushita Electric Works, Ltd., Osaka, Japan. His research interests include power electronics and wire communication engineering. Magnetics Society of Japan member.

Shogo Watanabe

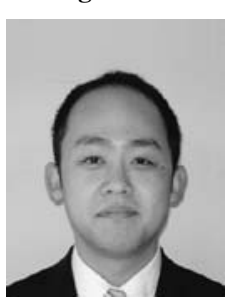

(Non-member) was born in Tokyo, Japan, in 1982. He received his B. S. and M.S. degrees in information engineering from the Kyushu Institute of Technology, Fukuoka, in 2004 and 2006, respectively. Now, he is an employee in Matsushita Electric Works, Ltd., Osaka, Japan.
Masaichi Fukunaga (Non-member) was born in Osaka, Japan, in 1955. He received his B. S. degree from Doshisha University, Kyoto, Japan, in 1978. He is currently a General Manager at New Product Technologies Development Department in Matsushita Electric Works, Ltd., Osaka, Japan. 